\title{
A novel class of tRNA-derived small RNAs extremely en- riched in mature mouse sperm
}

Cell Research (2012) 22:1609-1612. doi:10.1038/cr.2012.141; published online 9 October 2012

\section{Dear editor,}

The discovery of sperm-borne RNAs (mRNAs and small non-coding RNAs) has opened the possibility of additional paternal contributions aside from providing the DNA [1]. It has been reported that the incoming sperm can provide information for its host egg cytoplasm, which functionally influences the order of cell division [2], possibly via delivering RNAs. Indeed, the sperm-borne miRNA and mRNA have been demonstrated as active players in early embryo development [3] and transgenerational epigenetic inheritance [4]. However, given the diversity of small RNA classes (miRNA, endosiRNA, piRNA, etc.) generated during spermatogenesis, the contents and profiles of the small RNA population carried by mature sperm remain undefined. In the present study, we isolated mature sperm from the cauda epididymis of adult male mice (Supplementary information, Data S1). The purity of sperm was $>99 \%$ as evaluated by microscopy and was confirmed by RT-PCR analyses of different biomarkers (Supplementary information, Figure S1A and S1B). The RNA extracted from mature sperm, adult testis, and uterus were processed for small RNA ( $<40 \mathrm{nt})$ deep sequencing (Supplementary information, Figure S1C, S1D, S1E and Data S1). The total small RNA reads and genome-mapping statistic data (Supplementary information, Table S1) showed an abundance of small RNAs carried by mature sperm. The overall length distribution of small RNAs (Figure 1A) revealed that the dominant reads from mature sperm were at 29-34 nt, slightly different from adult testis (26-32 $\mathrm{nt})$, and distinct from uterus (21-23 nt). The majority of the 26-32 nt small RNAs in mouse testis are piwi-interacting RNAs (piRNAs), which are actively involved in retrotransposon silencing that protects the integrity of the genome [5]. As it was initially suggested that piRNAs are absent in the cauda epididymis [6] and that mammalian PIWI proteins (MILI, MIWI, MIWI2) are not expressed in mature sperm [5], the abundant existence of
29-34 nt small RNAs in the mature sperm is somewhat surprising to us and suggests that they might be different from the well-known piRNA population from testis. Further analysis has revealed a distinct signature for these mature-sperm-enriched small RNAs, which represent a novel class of abundantly expressed small RNAs that can be grouped into distinct families. The small RNAs within each family showed identical $5^{\prime}$ sequences and only differed at their distal 3' ends (Figure 1B and Supplementary information, Figure S2), suggesting that they are derived from the same precursor sequence. Particularly, two of these small RNA families were extremely enriched, which comprised $38.19 \%$ (family-1) and $19.14 \%$ (family-2) of all small RNA reads, respectively (Figure 1B), and together they accounted for the majority of the 30-34 nt small RNA population (Figure 1C).

To further characterize these mature-sperm-enriched small RNAs, we performed BLAT searches for the top two abundant families against the mouse genomic databases (mm9). As shown in Figure 1D, these small RNAs are located at multiple sites on the genome, with several clusters on chromosomes 1, 8 and 13. Most strikingly, each of these genomic locations corresponds to a tRNA locus (Figure 1D). By further comparing with the genomic tRNA database, we found that each of these small RNA families unanimously matches to the $5^{\prime}$ half of a specific tRNA, with cleavage sites located preferentially at the anticodon loop (30-34 nt from the 5' end), as illustrated for families 1-2 (Figure 1E and 1F) and for families 3-7 (Supplementary information, Figure S2). Their ultra-high enrichment (Figure 1G) and the preferential cleavage sites and length distributions strongly suggested that these small RNAs are not generated randomly by tRNA degradation, but under strict cleavage regulations. As these small RNAs are highly enriched in mature sperm and are derived from tRNAs, we termed them "mature-sperm-enriched tRNA-derived small RNAs" (mse-tsRNAs).

Indeed, recent evidence has demonstrated that tRNA- 


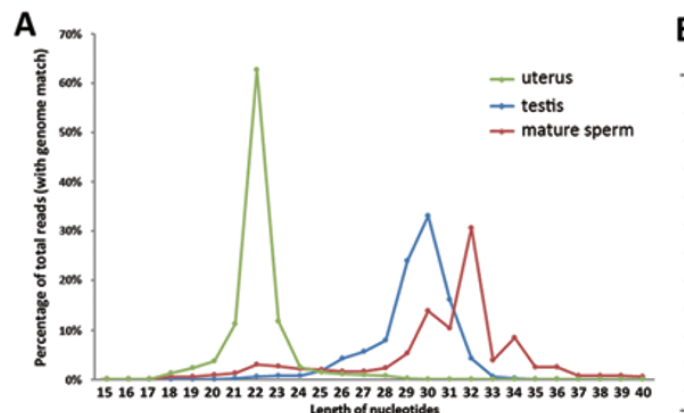

\begin{tabular}{|c|c|c|c|c|c|}
\hline B & Sequence $\left(5^{\prime}-3^{\prime}\right)$ & atth b & & Reads(\%) & \\
\hline & small RNA family-1 & & Sperm & Testis & Uterus \\
\hline TCCC & CTGGTGGTCTAGTGGTTAGGATTC & 27 & $197(0.00 \%)$ & $26(0.00 \%)$ & $18(0.00 \%)$ \\
\hline TCCO & ETGGTGGTCTAGTGGTTAGGATTCG & 28 & $823(0.01 \%)$ & $51(0.00 \%)$ & $26(0.00 \%)$ \\
\hline TCCO & CTGGTGGTCTAGTGGTTAGGATTCGG & 29 & $25806(0.30 \%)$ & $1741(0.01 \%)$ & $51(0.00 \%)$ \\
\hline $\mathrm{TCCC}$ & GTGGTGGTCTAGTGGTTAGGATTCGGC & 30 & $94632(1.09 \%)$ & $105764(0.60 \%)$ & $5(0.00 \%)$ \\
\hline $\mathrm{TCCO}$ & GTGGTGGTCTAGTGGTTAGGATTCGGCG & 31 & $342974(3.93 \%)$ & $5677(0.03 \%)$ & $4(0.00 \%)$ \\
\hline TCCC & GTGGTGGTCTAGTGGTTAGGATTCGGCGC & 32 & $2383402(27.33 \%)$ & $2312(0.01 \%)$ & $3(0.00 \%)$ \\
\hline TCCC & CTGGTGGTCTAGTGGTTAGGATTCGGCGCT & 33 & $152843(1.75 \%)$ & $246(0.00 \%)$ & $1(0.00 \%)$ \\
\hline TCCC & GTGGTGGTCTAGTGGTTAGGATTCGGCGCTC & 34 & $85609(0.98 \%)$ & $196(0.00 \%)$ & $0(0.00 \%)$ \\
\hline TCCC & ETGGTGGTCTAGTGGTTAGGATTCGGCGCTCT & 35 & 100978(1.16\%) & $27(0.00 \%)$ & $0(0.00 \%)$ \\
\hline TCCO & CTGGTGGTCTAGTGGTTAGGATTCGGCGCTCTC & 36 & $142427(1.63 \%)$ & $11(0.00 \%)$ & $0(0.00 \%)$ \\
\hline $\mathrm{TCCC}$ & CTGGTGGTCTAGTGGTTAGGATTCGGCGCTCTCA & 37 & $350(0.00 \%)$ & $0(0.00 \%)$ & $0(0.00 \%)$ \\
\hline $\operatorname{TCCO}$ & CTGGTGGTCTAGTGGTTAGGATTCGGCGCTCTCAC & 38 & $249(0.00 \%)$ & $0(0.00 \%)$ & $0(0.00 \%)$ \\
\hline & small RNA family-2 & & 3330290(38.19\%) & $116051(0.66 \%)$ & $108(0.00 \%)$ \\
\hline GCA & TTGGTGGTTCAGTGGTAGAATT & 25 & $240(0.00 \%)$ & $1(0.00 \%)$ & $13(0.00 \%)$ \\
\hline GCA & TTGGTGGTTCAGTGGTAGAATTC & 26 & $464(0.01 \%)$ & $54(0.00 \%)$ & $42(0.00 \%)$ \\
\hline GCA & TTGGTGGTTCAGTGGTAGAATTCT & 27 & $2733(0.03 \%)$ & $60.00 \%)$ & $7(0.00 \%)$ \\
\hline GCA & TTGGTGGTTCAGTGGTAGAATTCTC & 28 & $25877(0.30 \%)$ & $16(0.00 \%)$ & $2(0.00 \%)$ \\
\hline GCA & TTGGTGGTTCAGTGGTAGAATTCTCG & 29 & $67765(0.78 \%)$ & $11(0.00 \%)$ & $0(0.00 \%)$ \\
\hline GCA & TTGGTGGTTCAGTGGTAGAATTCTCGC & 30 & $643057(7.37 \%)$ & $15(0.00 \%)$ & $0(0.00 \%)$ \\
\hline GCA & TTGGTGGITCAGTGGTAGAATTCTCGCC & 31 & $250784(2.88 \%)$ & $9(0.00 \%)$ & $0(0.00 \%)$ \\
\hline GCA & TTGGTGGTTCAGTGGTAGAATTCTCGCCT & 32 & $138846(1.59 \%)$ & $4(0.00 \%)$ & $1(0.00 \%)$ \\
\hline GCA & TTGGTGGTTCAGTGGTAGAATTCTCGCCTG & 33 & $51980(0.60 \%)$ & $11(0.00 \%)$ & $0(0.00 \%)$ \\
\hline GCA & TTGGTGGTTCAGTGGTAGAATTCTCGCCTGC & 34 & $472739(5.42 \%)$ & $104(0.00 \%)$ & $0(0.00 \%)$ \\
\hline GCA & TTGGTGGTTCAGTGGTAGAATTCTCGCCTGCC & 35 & $13972(0.16 \%)$ & $38(0.00 \%)$ & $0(0.00 \%)$ \\
\hline GCA & TTGGTGGTTCAGTGGTAGAATTCTCGCCTGCCA & 36 & $320(0.00 \%)$ & $0(0.00 \%)$ & $0(0.00 \%)$ \\
\hline
\end{tabular}

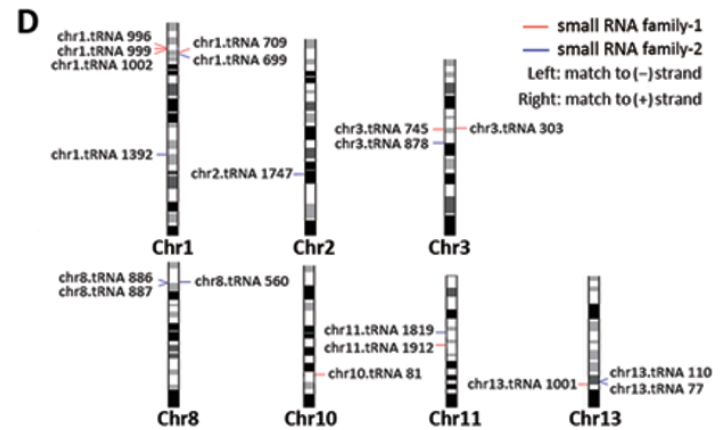

E ICCCTGGTGGTCTAGTGGTAGGATTCGGCGCTCTCACCGCCGCGGCCCGGGTICGATTCCCGGTCAGGGAA
TCCCTGGTGGTCTAGTGGTTAGGATTCGGCGCTCTCAC (38nt)
$\vdots$
TCCCTGGTGGTCTAGTGGTTAGGATTCGGCGC (32nt)
$\vdots$
TCCCTGGTGGTCTAGTGGTTAGGATTC (27nt)
The mse-tsRNA family-1 is derived from tRNA (Glu)

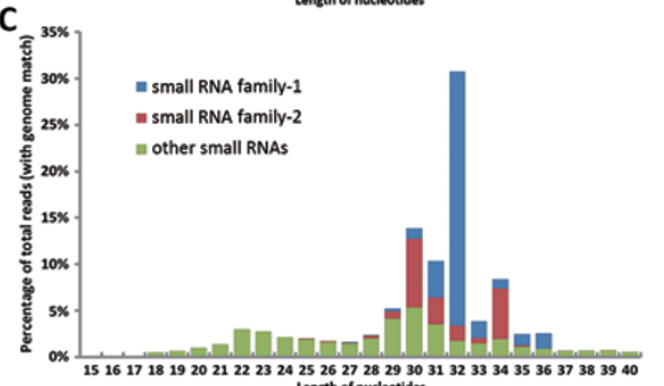

F GCATTGGTGGTTCAGTGGTAGAATTCTCGCCTGCCACGCGGGAGGCCCGGGTTNGNTTCCCGGCCAATGCA

G

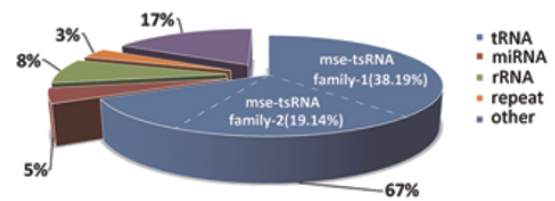

GCATTGGTGGTTCAGTGGTAGAATTCTCGCCTGCCA (36nt)

:

GCATTGGTGGTTCAGTGGTAGAATTCTCGC (30nt)

$\vdots$

GCATTGGTGGTTCAGTGGTAGAATT (25nt)

The mse-tsRNA family-2 is derived from tRNA (Gly)

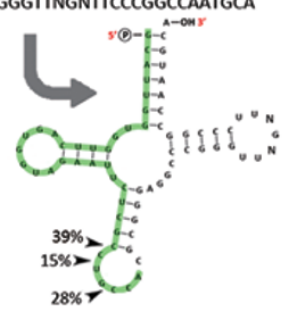

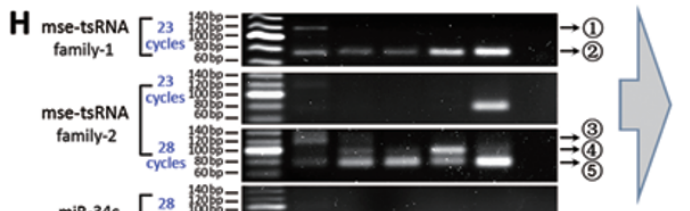

Primer for mse-tsRNA family-1 Primer for mse-tsRNA family-2 Primer for miR-34c

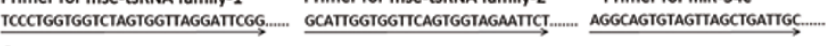

(1) TCCCTGGTGGTCTAGTGGITAGGATTCGGCGCTCTCACCGCCGCGGCCCGGGTTCGATTCCCGGTCAGGGAACC (69-74nt)

(2) TCCCTGGTGGTCTAGTGGTTAGGATTCGGCGCTC(29-34nt)

(3) GCATIGGTGGTICAGTGGTAGAATTCTCGCCTGCCACGCGGGAGGCCCGGGTICGATTCCCGGCCAATGCACC (73nt)

(4) GCATGGTGGTTCAGTGGTAGAATTCTCGCCTGCCACGCGGGAGGCCCGGGT(S2nt)

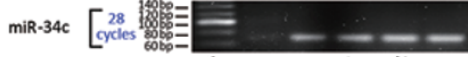

(5) GCATIGGTGGTICAGTGGTAGAATTCTCGCCTGC (28-34nt)

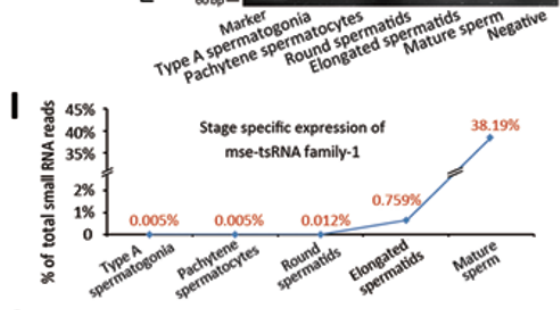

K

Intact sperm Purified sperm heads

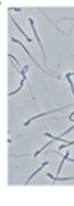

Purified sperm heads

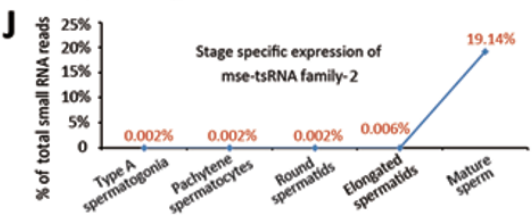

L

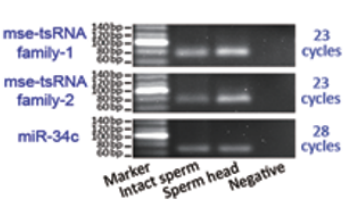

\begin{tabular}{|lcc} 
Species & $\begin{array}{c}\text { mse-tsRNA } \\
\text { family-1 }\end{array}$ & $\begin{array}{c}\text { mse-tsRNA } \\
\text { family-2 }\end{array}$ \\
\hline Arabidopsis thaliana & - & - \\
\hline Caenorhabditis elegans & - & - \\
\hline Drosophila melanogaster & - & - \\
\hline Petromyzon marinus & + & + \\
\hline Danio rerio & + & + \\
\hline Xenopus tropicalis & + & + \\
- Anolis carolinensis & + & + \\
Meleagris gallopavo & + & + \\
\hline - Mrnithorhynchus anatinus & + & + \\
Mammalia & + & +
\end{tabular}

$\mathbf{N}$

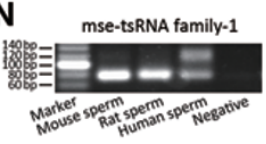

mse-tsRNA family-2

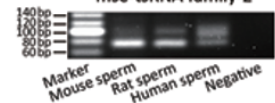

Cell Research | Vol 22 No 11 | November 2012 
derived RNA fragments are biologically functional [7], and their production could be induced under various stress conditions (physical or chemical stress) by specific RNase [8]. The mature sperm is produced from testicular spermatogenesis followed by maturation during passage through the epididymis. The physiological condition and specific enzyme governing mse-tsRNAs production and accumulation in mature sperm are currently unknown.

To monitor the biogenesis of mse-tsRNAs during sperm formation, we next analyzed purified mouse testicular spermatogenic cells (type A spermatogonia, pachytene spermatocytes, round spermatids and elongated spermatids) and mature sperm. We found that the levels of mse-tsRNA family-1 and -2 were relatively constant during the early stages of spermatogenesis, and were substantially increased at late- (family-1) or postspermatogenesis (family-2) (Figure 1H). The RT-PCR results were further confirmed by analyzing small RNA deep-sequencing data obtained from purified mouse testicular spermatogenic cells [9] and mature sperm (Figure $1 \mathrm{I}$ and $1 \mathrm{~J}$ ). By analyzing the PCR product size followed by product sequencing, we could find both mse-tsRNAs and their tRNA precursors, supporting the hypothesis that the mse-tsRNAs are derived from tRNA cleavage (Figure $1 \mathrm{H})$. Interestingly, we consistently observed a sequence (52 nt) in mse-tsRNA family-2 PCR products, which is shorter than the expected length of intact tRNA and mapped to its $5^{\prime}$ portion (Figure $1 \mathrm{H}$ ), suggesting the involvement of a two-step cleavage of tRNA in generating mse-tsRNA family-2. The increase of mse-tsRNA family-1 and -2 seems not to correlate with the expression of mammalian PIWI proteins (MILI, MIWI, MIWI2) [5], suggesting that they might not be closely related to the piRNAs, and their ultra-high enrichment in mature sperm might be due to specific tRNA cleavage and/or selective accumulation of cleavage products at late- or postspermatogenesis (such as during epididymal transition).
The underlying mechanisms are currently unknown.

We also analyzed the relative expression of miR-34c by RT-PCR as a quality control, as its expression in spermatogenic cells and mature sperm have been previously reported $[3,10]$. As shown in Figure 1H, our results were consistent with previous reports that miR-34c was almost absent in spermatogonia, but was highly expressed from pachytene spermatocytes and continued to be highly expressed in spermatids [10] and mature sperm [3]. Note that miR-34c expression in mature sperm is much less than that of mse-tsRNA family-1 and -2 , as shown by the RT-PCR results and by the reads number from our miRNA profiling database (Supplementary information, Figure S3).

It is important to determine whether the sperm-borne mse-tsRNAs are located in sperm head, which could indicate their potential delivery into oocytes at fertilization. Using established methods to isolate purified sperm heads, we demonstrated that mse-tsRNAs are abundantly localized in the purified sperm head (Figure $1 \mathrm{~K}$ and $1 \mathrm{~L}$ ), suggesting that they could be delivered into oocytes at fertilization. We next analyzed the expression levels of mse-tsRNA family-1 in oocytes, zygotes and parthenogenetically activated oocytes. Surprisingly, quantitative RT-PCR analysis revealed that the level of mse-tsRNA family-1 in zygotes is significantly lower than that in the oocytes and parthenogenetically activated oocytes (Supplementary information, Figure S4). These results might suggest a fertilization-triggered usage/consumption of mse-tsRNAs, which may reflect a functional role for mse-tsRNAs in early embryo events.

As the mse-tsRNAs are derived from their tRNA templates, their sequence conservation could simply reflect the evolutionary conservation of their tRNA precursors. As shown in the evolutionary conservation analysis (Figure $1 \mathrm{M}$ and Supplementary information, Table S2), the tRNAs generating mse-tsRNA families 1-7 are highly

Figure 1 Identification and characterization of tRNA-derived small RNAs in mature mouse sperm. (A) Length distributions of small RNAs in uterus, testis and mature sperm. (B) Alignments and statistics of small RNA family-1 and -2 in mature sperm, testis and uterus; the top three enriched RNAs for each family were highlighted. (C) Small RNA family-1 and -2 comprised the majority of 30-34 nt small RNA population. (D) Chromosome locations of small RNA family-1 and -2. (E, F) Illustrations showing that mse-tsRNA family-1 (E) and mse-tsRNA family-2 (F) are derived from $5^{\prime}$ halves of tRNA ${ }^{\text {Glu }}$ and tRNA ${ }^{\text {Gly }}$ respectively; top three cleavage sites for each tRNA were marked by arrow heads. (G) Catalogue of small RNA populations in mature mouse sperm. (H) RT-PCR analyses of mse-tsRNA family-1, -2 and miR-34c in purified spermatogenic cells and mature sperm, followed by product sequencing. The variable nucleotides were marked by shade. Samples for RT-PCR were equally loaded by calibration of RNA concentration. Similar results were obtained in 3 independent experiments. (I, J) Percentages of mse-tsRNA family-1 (I) and -2 (J) reads from deep-sequencing datasets of purified spermatogenic cells and mature sperm. (K) Representative photos of intact sperm and purified sperm heads. (L) RT-PCR analyses of mse-tsRNA family-1, -2 and miR$34 \mathrm{c}$ in intact sperm and purified sperm heads. (M) Evolutionary tree showing that the tRNA precursors of mse-tsRNA family-1 and -2 are highly conserved in vertebrate species. (N) RT-PCR analyses of mse-tsRNA family-1 and -2 in mature sperm from mouse, rat and human. 
conserved in vertebrate species, from fish to mammals, but absent from the worms, flies and plants. Indeed, besides our reported data for mouse sperm, existing small RNA datasets have shown that the mse-tsRNA family-1 is among the most highly expressed small RNA sequences in zebra fish testis (NCBI GEO Datasets: GSM830247) [11] and human sperm (NCBI GEO Datasets: GSM530235) [12], supporting the spermatozoal expression of mse-tsRNAs in a wide range of species. The expression of mse-tsRNA family-1 and -2 in mature sperm from mouse, rat and human were further analyzed using RT-PCR and confirmed by product sequencing (Figure 1N). These results suggest that mse-tsRNAs might serve as an ancient paternal element with evolutionarily conserved functions.

Taken together, the present study revealed a previously hidden layer of sperm-borne small RNAs, identifying a novel class of tRNA-derived mse-tsRNAs with ultrahigh accumulation in mature sperm. The biogenesis and function of these mse-tsRNAs are interesting topics that warrant future investigations.

\section{Acknowledgments}

This research was supported by National Basic Research Program of China (2011CB944401 and 2011CB710905), Strategic Priority Research Program of the Chinese Academy of Sciences (XDA 01010202), National Natural Science Foundation of China (31200879), and Sciences Knowledge Innovation Program of the Chinese Academy of Sciences (KSCX2-EW-R-06).

Hongying Peng ${ }^{1, *}$, Junchao Shi, *, Ying Zhang ${ }^{1}$, He Zhang ${ }^{1}$, Shangying Liao', Wei $\mathrm{Li}^{1,2}$, Li Lei ${ }^{1,2}$, Chunsheng Han ${ }^{1}$, Lina Ning ${ }^{1}$, Yujing Cao', Qi Zhou', Qi Chen ${ }^{1}$, Enkui Duan ${ }^{1}$

${ }^{I}$ State Key Laboratory of Reproductive Biology, Institute of Zoology, Chinese Academy of Sciences, Beijing 100101, China; ${ }^{2}$ Graduate Universty of Chinese Academy of Sciences, Beijing 100049, China

*These two authors contributed equally to this work. Correspondence: Enkui Duan ${ }^{\mathrm{a}}$, Qi Chen ${ }^{\mathrm{b}}$

${ }^{\mathrm{a}}$ Tel: +86-10-64807182;

E-mail: duane@ioz.ac.cn
${ }^{\mathrm{b}}$ Tel: +86-10-64807182

E-mail: chenqi@ioz.ac.cn

\section{References}

1 Krawetz SA. Paternal contribution: new insights and future challenges. Nat Rev Genet 2005; 6:633-642.

2 Piotrowska K, Zernicka-Goetz M. Role for sperm in spatial patterning of the early mouse embryo. Nature 2001; 409:517521

3 Liu WM, Pang RT, Chiu PC, et al. Sperm-borne microRNA$34 \mathrm{c}$ is required for the first cleavage division in mouse. Proc Natl Acad Sci USA 2012; 109:490-494.

4 Rassoulzadegan M, Grandjean V, Gounon P, et al. RNA-mediated non-mendelian inheritance of an epigenetic change in the mouse. Nature 2006; 441:469-474.

5 Thomson T, Lin H. The biogenesis and function of PIWI proteins and piRNAs: progress and prospect. Annu Rev Cell Dev Biol 2009; 25:355-376. .

6 Grivna ST, Beyret E, Wang Z, Lin H. A novel class of small RNAs in mouse spermatogenic cells. Genes Dev 2006; 20:1709-1714.

7 Lee YS, Shibata Y, Malhotra A, Dutta A. A novel class of small RNAs: tRNA-derived RNA fragments (tRFs). Genes Dev 2009; 23:2639-2649.

8 Yamasaki S, Ivanov P, Hu GF, Anderson P. Angiogenin cleaves tRNA and promotes stress-induced translational repression. $J$ Cell Biol 2009; 185:35-42.

9 Gan $\mathrm{H}$, Lin X, Zhang Z, et al. piRNA profiling during specific stages of mouse spermatogenesis. RNA 2011; 17:1191-1203.

10 Liang X, Zhou D, Wei C, et al. MicroRNA-34c enhances murine male germ cell apoptosis through targeting ATF1. PLoS One 2012; 7:e33861.

11 Kamminga LM, Luteijn MJ, den Broeder MJ, et al. Hen1 is required for oocyte development and piRNA stability in zebrafish. Embo J 2010; 29:3688-3700.

12 Krawetz SA, Kruger A, Lalancette C, et al. A survey of small RNAs in human sperm. Hum Reprod 2011; 26:3401-3412.

(Supplementary information is linked to the online version of the paper on the Cell Research website.)

This work is licensed under the Creative Commons Attribution-NonCommercial-No Derivative Works 3.0 Unported License. To view a copy of this license, visit http:// creativecommons.org/licenses/by-nc-nd/3.0 